\title{
Long-term outcomes of tracheobronchial stenosis due to tuberculosis (TSTB) in symptomatic patients: airway intervention vs. conservative management
}

\author{
Ken Cheah Hooi Lee ${ }^{1,2}$, Shera Tan ${ }^{3}$ Junyang Ken Goh ${ }^{1,2}$, Anne Ann Ling Hsu ${ }^{1,2}$, Su Ying Low ${ }^{1,2}$ \\ ${ }^{1}$ Department of Respiratory and Critical Care Medicine, Singapore General Hospital, Singapore, Singapore; ${ }^{2}$ Lung Center, Duke-National \\ University Singapore SingHealth, Singapore, Singapore; ${ }^{3}$ Tuberculosis Control Unit, Tan Tock Seng Hospital, Singapore, Singapore \\ Contributions: (I) Conception and design: AAL Hsu, SY Low; (II) Administrative support: S Tan, JK Goh; (III) Provision of study materials or \\ patients: AAL Hsu; (IV) Collection and assembly of data: AAL Hsu, S Tan, KCH Lee; (V) Data analysis and interpretation: All authors; (VI) \\ Manuscript writing: All authors; (VII) Final approval of manuscript: All authors. \\ Correspondence to: Dr. Ken Cheah Hooi Lee. Department of Respiratory and Critical Care Medicine, 20 College Road, Singapore 169856, Singapore. \\ Email: ken.lee.c.h@singhealth.com.sg.
}

Background: Patients with tracheobronchial stenosis due to tuberculosis (TSTB) have a variable clinical course and response to treatment including airway intervention. There are no clear guidelines on the best approach to manage such patients. This study examines long-term outcomes of patients with TSTB and factors associated with recurrent symptoms or need for repeat airway intervention following initial bronchoscopic intervention.

Methods: This is a retrospective analysis of patients with TSTB over an 18-year period. Symptoms, radiological, bronchoscopic findings, airway interventions and complications were obtained. Multivariate logistic regression analysis was performed to identify factors predictive of recurrence of symptoms or need for repeat airway intervention.

Results: A total of 131 patients with mean age $50 \pm 18$ years and median follow-up 5 (interquartile range, 2-10) years were included. Nineteen $(29.7 \%)$ patients underwent balloon dilatation alone, $22(34.4 \%)$ had additional resection or stenting, and 19 (29.7\%) underwent all 3 procedures. Among patients conservatively managed, a larger proportion (55.6\%) of patients with no recurrence of symptoms had active TB. Patients who developed recurrent symptoms had longer symptom duration (12 vs. 3 months, $\mathrm{P}=0.025)$, and more had recurrent lower respiratory tract infections ( $50 \%$ vs. $5.6 \%, \mathrm{P}=0.003)$, lung collapse $(90.0 \%$ vs. $41.7 \%$, $\mathrm{P}=0.011)$ or linear fibrosis $(70.0 \%$ vs. $30.6 \%, \mathrm{P}=0.033)$ compared to those without recurrent symptoms. Bronchomalacia $(\mathrm{OR}=17.18$; 95\% CI, 3.43-86.18) and prior bronchoscopic airway resection $(\mathrm{OR}=4.97$; 95\% CI, 1.20-20.60) were strongly associated with the recurrence of symptoms or need for repeat airway intervention on multivariate logistic regression analysis.

Conclusions: Bronchomalacia and prior bronchoscopic airway resection are associated with the recurrence of symptoms despite airway intervention. Patients who are diagnosed with TSTB early in the course of active TB may be conservatively managed.

Keywords: Tuberculosis; tracheobronchial stenosis; bronchomalacia; airway intervention

Submitted Jan 25, 2020. Accepted for publication May 19, 2020.

doi: 10.21037/JTD-20-670

View this article at: http://dx.doi.org/10.21037/JTD-20-670 


\section{Introduction}

Endobronchial tuberculosis has been reported in up to $40 \%$ of patients with pulmonary tuberculosis (pTB) based on studies performed prior to the availability of anti-tuberculous therapy $(1,2)$. Despite improved living conditions and better access to healthcare which have led to an overall decrease in TB incidence, endobronchial TB and tracheobronchial stenosis due to TB (TSTB) remain a significant problem. The incidence of endobronchial TB or TSTB ranges between $6 \%$ and $50 \%$ across different centres (3-8). A high index of suspicion and computed tomography (CT) of the chest or bronchoscopy may be needed to confirm the diagnosis (9-11).

The clinical course of TSTB is highly variable (12). Some patients suffer from debilitating symptoms while others remain asymptomatic. Bronchoscopic interventions such as balloon dilatation and stenting may be performed for symptom relief (13-15). However, some patients have persistent or recurrent symptoms despite intervention and may even suffer from complications of the procedure $(16,17)$. A conservative approach on the other hand may lead to progressive airway stenosis and development of complications like bronchiectasis, recurrent pneumonia or lung collapse in the longer term.

To date there are no consensus guidelines on the best approach to manage patients with TSTB. This study provides an overview of the long-term outcomes of patients with TSTB in a tertiary care hospital and examines the factors associated with recurrence of symptoms or need for airway re-intervention following bronchoscopic procedures performed for TSTB. This would aid physicians in the selection of patients who are more likely to benefit from airway intervention.

\section{Methods}

We performed a retrospective analysis of patients with TSTB resulting from active or past TB treated at the Singapore General Hospital, a tertiary-care universityaffiliated hospital, from September 1998 to December 2015. Eligible patients were selected from a database using the following inclusion criteria: patients with TSTB diagnosed on bronchoscopy secondary to active or past tuberculosis. We excluded patients with tracheobronchial stenosis secondary to etiologies other than tuberculosis.

Active pTB was defined by current microbiological evidence of TB at the time of diagnosis of tracheobronchial stenosis. Past pTB was defined by a documented history or previous microbiological positivity of pTB. Local symptoms were defined by the presence of cough, dyspnoea or haemoptysis. Systemic symptoms were defined by the presence of fever or loss of weight. The degree of airway stenosis was classified into mild, moderate, severe and pinhole (Figure S1) based on 25\%, 25-50\%, 50-90\% and $>90 \%$ occlusion of the affected airway diameter respectively.

All symptomatic patients were offered airway interventions including balloon dilatation, airway stenting and luminal resection. Patients who were deemed unfit or declined airway interventions were conservatively managed. Those with active pTB were commenced on anti-tuberculous therapy and monitored for response to treatment before they were considered for further interventions. All patents underwent flexible bronchoscopy using the Olympus therapeutic bronchoscope with a minimum $2.0 \mathrm{~mm}$ working channel. Rigid bronchoscopy was performed using the Dumon rigid bronchoscope under general anaesthesia. Only silicone stents were deployed. Luminal resection was performed using the Neodymiumdoped yttrium aluminium garnet (Nd-YAG) laser.

Clinical case-notes and electronic medical records of all patients were reviewed. Demographics, symptoms, radiological, bronchoscopic findings, airway interventions and complications were recorded.

Continuous variables (age, degree of airway stenosis and duration of symptoms) were presented as mean \pm standard deviation (SD) or median [interquartile range (IQR)] and were compared using $t$-tests or Mann-Whitney $\mathrm{U}$ tests. Categorical variables (gender, activity of pTB and number of airway stenoses) were presented as number (percentage) and studied using Pearson's chi-square or the Fisher's exact test where appropriate. Multivariate logistic regression analysis was performed to identify factors that were predictive of recurrent symptoms or need for reintervention following the initial airway intervention. All analyses were performed using SPSS (SPSS, version 17.0; SPSS Inc., Chicago, IL, USA). A P value of less than 0.05 was used to indicate statistical significance. This was a retrospective case study. Ethics approval for this study was obtained from the SingHealth Institutional Review Board (201501-00097) without explicit consent required from the participants.

\section{Results}

One hundred and thirty-one patients were included in the 
Table 1 Patient characteristics $(n=131)$

\begin{tabular}{|c|c|}
\hline Characteristics & $\begin{array}{c}\text { Number } \\
\text { (proportion \%) }\end{array}$ \\
\hline Mean age \pm SD (years) & $50 \pm 18$ \\
\hline \multicolumn{2}{|l|}{ Gender } \\
\hline Male & $34(26.0)$ \\
\hline Female & $97(74.0)$ \\
\hline \multicolumn{2}{|l|}{ Activity of pTB } \\
\hline Active pTB & $47(35.9)$ \\
\hline Past pTB & $84(64.1)$ \\
\hline Asymptomatic & $24(18.3)$ \\
\hline Symptomatic & $107(81.7)$ \\
\hline Local symptoms only & $78(59.5)$ \\
\hline Systemic symptoms only & $5(3.8)$ \\
\hline Local and systemic symptoms & $24(18.3)$ \\
\hline Conservative management & $46(43.0)$ \\
\hline Underwent airway intervention & $61(57.0)$ \\
\hline \multicolumn{2}{|l|}{ Follow up status } \\
\hline Still on follow-up & $24(18.3)$ \\
\hline Lost to follow-up & $32(24.4)$ \\
\hline Discharged & $75(57.3)$ \\
\hline Follow-up duration for all patients (years) & $5[2,10]$ \\
\hline \multicolumn{2}{|l|}{ Duration to diagnosis of TSTB } \\
\hline $\begin{array}{l}\text { Time from start of anti-TB } \\
\text { medication for active TB (months) }\end{array}$ & $3[1,7]$ \\
\hline Time from diagnosis of past pTB (years) & $15[8,25]$ \\
\hline
\end{tabular}

Data presented as $\mathrm{n}(\%)$ or median (interquartile range) unless otherwise indicated. pTB, pulmonary tuberculosis; TSTB, tracheobronchial stenosis due to tuberculosis.

study, of whom 107 were symptomatic. Sixty-one patients $(57 \%)$ underwent bronchoscopic airway intervention while the rest were conservatively managed for their symptoms (Table 1). Forty-seven patients had active pTB with a median duration of 3 months from start of anti-TB medication to diagnosis of TSTB. The median duration from diagnosis of TB to presentation with TSTB was 15 years. Twenty-four $(18.3 \%)$ patients were still on follow-up during the time of the study. Median follow-up duration was 5 years.

Lung collapse and consolidation were the most common radiological findings detectable on both chest radiographs
(CXR) and CT scans. Computed tomography scans identify more airway abnormalities compared to CXR. Nodular infiltrates were more common in patients with active pTB, while granulomas, airway abnormalities and lung collapse were more common among those with previous disease (Table 2).

All patients had airway stenosis on bronchoscopy. Nearly half had more than 1 stenotic site. Stenoses most frequently involved the left main bronchus (34.9\%) and the trachea $(17.2 \%)$. The majority of stenoses were severe, involving $>50 \%$ of the airway lumen. Pinhole stenoses were most common in the upper lobe bronchi (Table 3). The most common endobronchial abnormalities were fibrosis (56.5\%) (Figure S2A,B), airway distortion (34.4\%) (Figure S3) and bronchomalacia (29\%) (Figure S2C). Endobronchitis, ulceration, caseous material and nodular lesions (Figure S2D) were more common in patients with active pTB (Table 2). Airway fibrosis was seen in a significantly higher proportion of those with previously treated pTB compared to active disease $(67.9 \%$ vs. $36.2 \%, \mathrm{P}<0.001)$.

Among the 64 patients who underwent airway intervention, 3 were asymptomatic but had interventions performed for lung collapse noted on CT. Nineteen (29.7\%) patients underwent balloon dilatation alone, 22 (34.4\%) had additional resection or stenting, while 19 (29.7\%) underwent all 3 procedures. Half of the airway interventions involved stent insertion, with the majority having a single stent inserted. The most common site of stenting was the left main bronchus (63.4\%) (Table 4). Median stent diameter was $11 \mathrm{~mm}$ (IQR, 10-13 mm), and median length was $40 \mathrm{~mm}$ (IQR, 30-40 mm). One patient failed deployment with even the smallest $(10 \mathrm{~mm}$ diameter) silicone stent available due to difficult dilatation of a severely calcified bronchus.

Patients who underwent balloon dilatation or airway stenting had a median pre and post airway intervention diameter of $3 \mathrm{~mm}$ (IQR, 2-5 mm) and $10 \mathrm{~mm}$ (IQR, 8$11 \mathrm{~mm}$ ) respectively. Significant airway dilatation was achieved in the trachea, left and right main bronchus from pre-intervention diameter of $6 \mathrm{~mm}$ (IQR, 4-7 mm), $2 \mathrm{~mm}$ (IQR, 2-4 mm) and $4 \mathrm{~mm}$ (IQR, 2-5 mm) to $12 \mathrm{~mm}$ (IQR, 10-14 mm), $10 \mathrm{~mm}$ (IQR, 6-10 $\mathrm{mm}$ ) and $10 \mathrm{~mm}$ (IQR, $7-10 \mathrm{~mm}$ ) respectively after intervention. The stent-related complications (Figure S4) are shown in Table 5.

Information on the improvement of symptoms, CXR findings and spirometry after airway intervention was available for 43, 41 and 28 patients respectively. Of these, $40(93 \%)$ patients improved symptomatically, while 
Table 2 Comparison of active versus past pTB

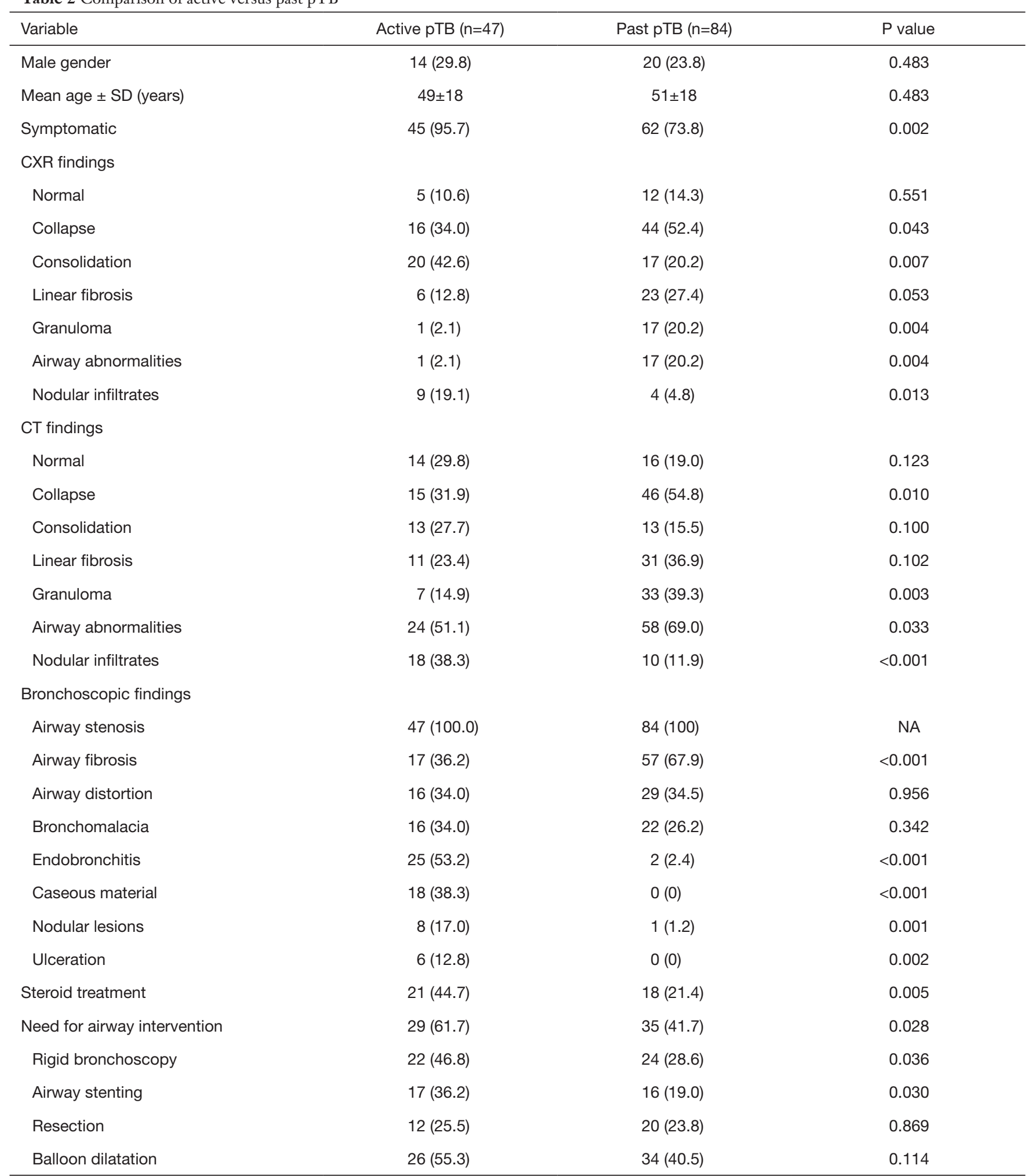

Data presented as $\mathrm{n}(\%)$ unless otherwise indicated. pTB, pulmonary tuberculosis; CT, computed tomography. 
Table 3 Site and severity of airway stenoses

\begin{tabular}{|c|c|c|c|c|}
\hline Site & \multicolumn{4}{|c|}{ Severity of stenosis } \\
\hline Trachea & $17(47.2)$ & $1(2.8)$ & $18(50.0)$ & $0(0)$ \\
\hline RULB & $0(0)$ & $3(12.0)$ & $12(48.0)$ & $10(40.0)$ \\
\hline RBI & $1(7.7)$ & $3(23.1)$ & 7 (53.8) & $2(15.4)$ \\
\hline RLLB & $0(0)$ & $0(0)$ & $4(100.0)$ & $0(0)$ \\
\hline LMB & $4(5.5)$ & $3(4.1)$ & $56(76.7)$ & $10(13.7)$ \\
\hline LULB & $1(3.8)$ & $3(11.5)$ & $14(53.8)$ & $8(30.8)$ \\
\hline LLLB & 0 & $1(16.7)$ & $4(66.6)$ & $1(16.7)$ \\
\hline
\end{tabular}

Data presented as $\mathrm{n}$ (\%). RMB, right main bronchus; RULB, right upper lobe bronchus; RBI, right bronchus intermedius; RMLB, right middle lobe bronchus; RLLB, right lower lobe bronchus; LMB, left main bronchus; LULB, left upper lobe bronchus; LLLB, left lower lobe bronchus.

Table 4 Airway interventions performed at the initial procedural session

\begin{tabular}{|c|c|}
\hline Procedure & Frequency \\
\hline \multicolumn{2}{|l|}{ Type of intervention $(n=64)$} \\
\hline Balloon dilatation only & $19(29.7)$ \\
\hline Stenting only & $2(3.0)$ \\
\hline Resection only & $1(1.6)$ \\
\hline Resection \& balloon dilatation & $11(17.2)$ \\
\hline Balloon dilatation \& stenting & $11(17.2)$ \\
\hline Resection \& stenting & $1(1.6)$ \\
\hline Resection, balloon dilatation \& stenting & $19(29.7)$ \\
\hline \multicolumn{2}{|l|}{ Number of stents inserted $(n=33)$} \\
\hline 1 stent & $27(81.8)$ \\
\hline 2 stents & $4(12.1)$ \\
\hline 3 stents & $2(6.1)$ \\
\hline \multicolumn{2}{|l|}{ Site of stent insertion $(n=33)$} \\
\hline Trachea only & $7(21.2)$ \\
\hline LMB only & $17(51.5)$ \\
\hline Distal trachea and LMB & $2(6.1)$ \\
\hline RMB only & $3(9.1)$ \\
\hline RBI only & $1(3.0)$ \\
\hline Trachea, LMB, RMB & $2(6.1)$ \\
\hline Failed attempt & $1(3.0)$ \\
\hline
\end{tabular}

Data presented as $\mathrm{n}$ (\%). LMB, left main bronchus; RMB, right main bronchus; RBI, right bronchus intermedius.
Table 5 Stent-related complications

\begin{tabular}{lc}
\hline Complication $(\mathrm{n}=20)$ & Frequency \\
\hline Restenosis & $15[75]$ \\
Granuloma & $15[75]$ \\
Stent migration & $5[25]$ \\
Infection & $4[20]$ \\
Mucous plugging & $7[35]$ \\
Need for re-intervention & $16[80]$ \\
\hline
\end{tabular}

Data presented as $\mathrm{n}[\%]$.

25 (61.0\%) and 24 (85.7\%) patients showed improvement on CXR and spirometry respectively. All 9 (6.9\%) patients who underwent surgery did well and were discharged.

Twenty-one out of the 67 patients (31\%) who were conservatively managed were asymptomatic. Among the symptomatic patients who were conservatively managed, a larger proportion of those without recurrence of symptoms had active TB and systemic symptoms at the time of diagnosis of TSTB (Table 6). On the other hand, patients who developed recurrent symptoms were found to have longer duration of symptoms (12 vs. 3 months, $\mathrm{P}=0.025$ ), and a larger proportion of them had recurrent lower respiratory tract infections $(50 \%$ vs. $5.6 \%, \mathrm{P}=0.003)$, CT findings of lung collapse $(90.0 \%$ vs. $41.7 \%, \mathrm{P}=0.011)$ or linear fibrosis $(70.0 \%$ vs. $30.6 \%, \mathrm{P}=0.033)$ compared to patients who had no recurrent symptoms.

Table 7 shows the outcomes of the patients who 
Table 6 Factors associated with recurrence of symptoms in patients who did not undergo airway intervention $(\mathrm{n}=46)$

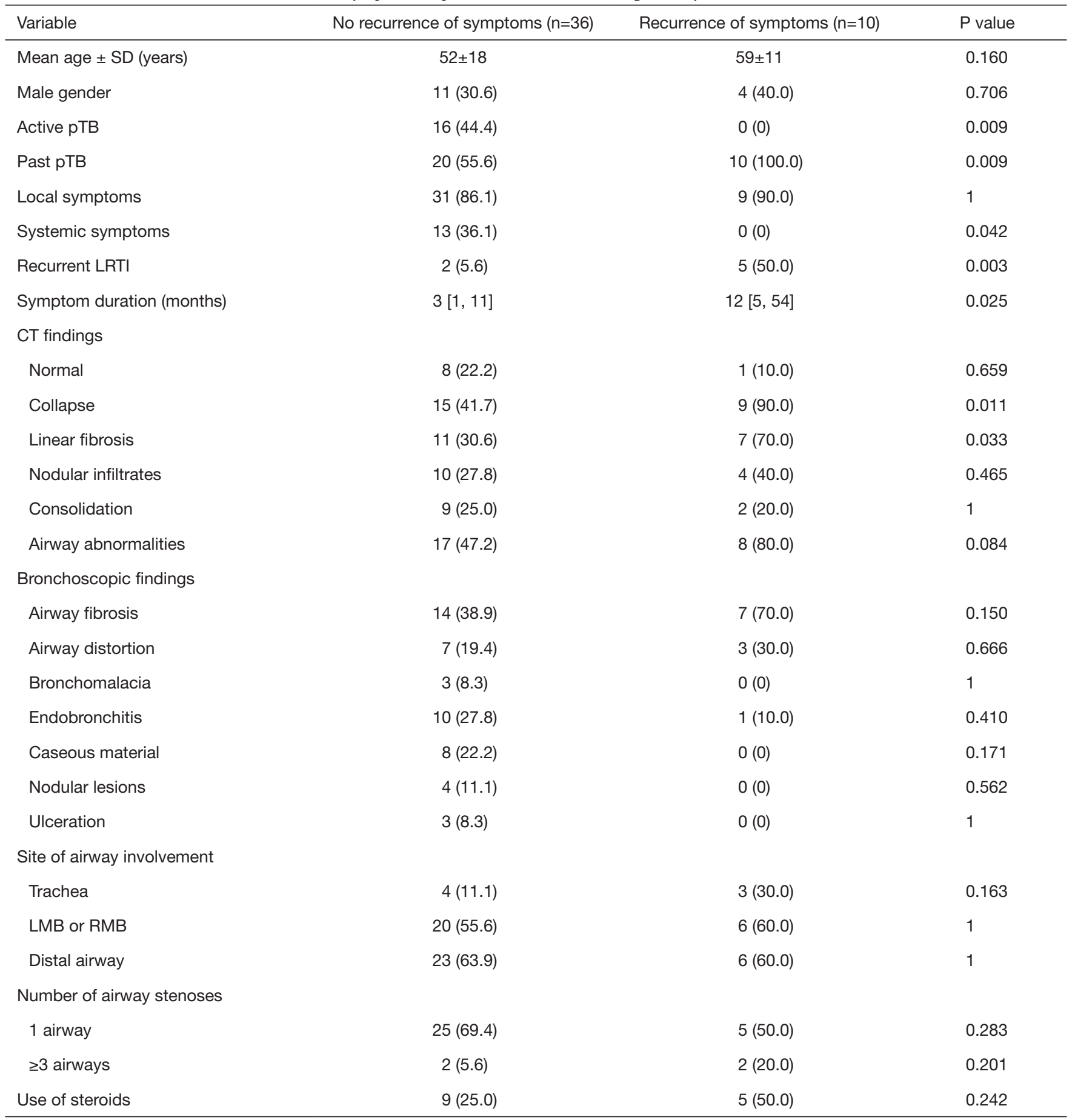

Data presented as $\mathrm{n}$ (\%) or median (interquartile range) unless otherwise indicated. pTB, pulmonary tuberculosis; CT, computed tomography; LRTI, lower respiratory tract infections; LMB, left main bronchus; RMB, right main bronchus. 
Table 7 Factors associated with recurrence of symptoms or need for re-intervention in patients who underwent airway intervention (n=61)

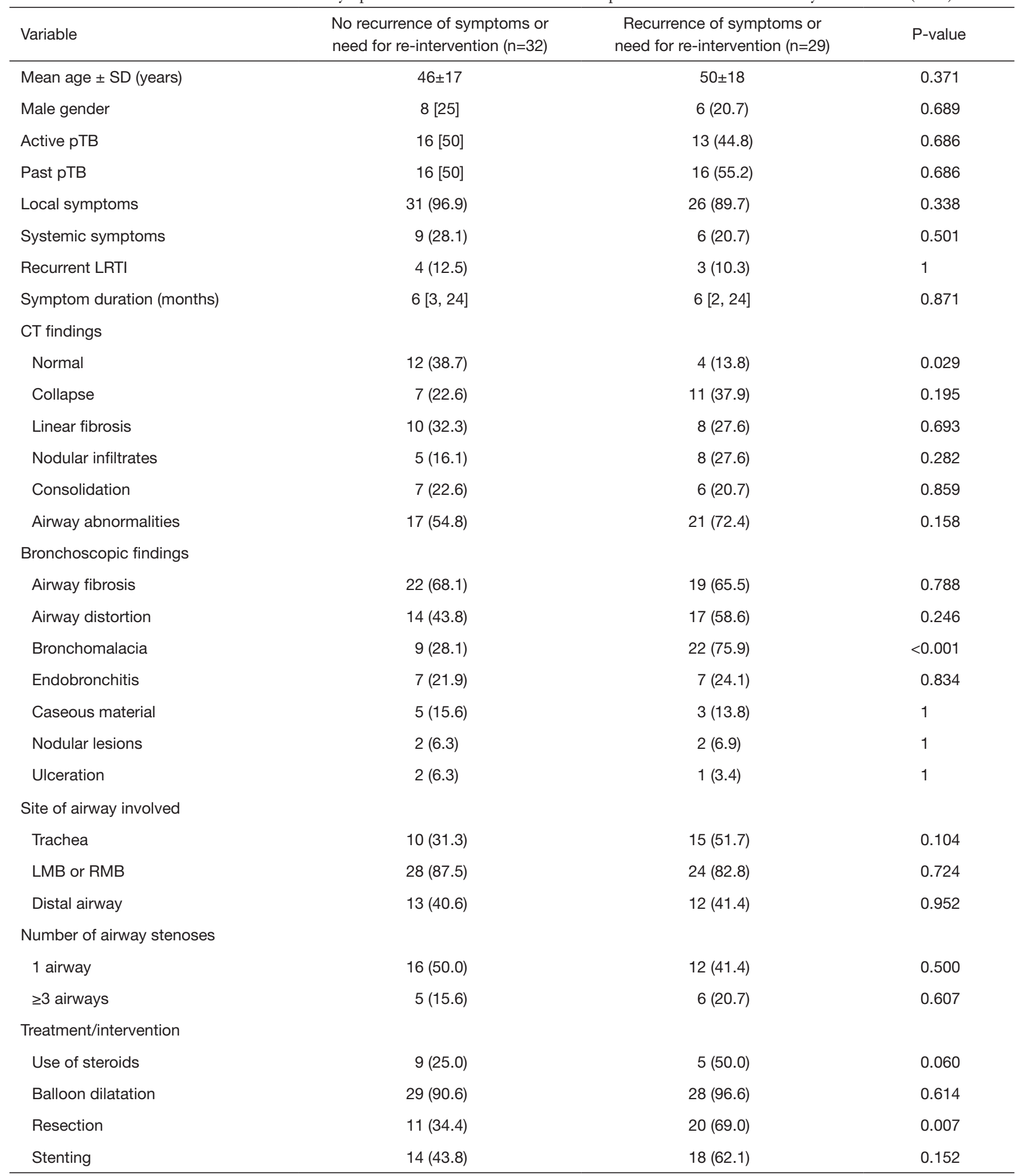

Data presented as $\mathrm{n}(\%)$ or median (interquartile range) unless otherwise indicated. pTB, pulmonary tuberculosis; CT, computed tomography; LRTI, lower respiratory tract infections; LMB, left main bronchus; RMB, right main bronchus. 
Table 8 Multivariate logistic regression analysis for factors associated with recurrence of symptoms or re-intervention in patients who underwent airway intervention $(\mathrm{n}=61)$

\begin{tabular}{lcc}
\hline Variable & Odds ratio (95\% Cl) & P value \\
\hline Normal CT scan & $0.087(0.014-0.534)$ & 0.008 \\
Bronchomalacia on bronchoscopy & $17.179(3.425-86.179)$ & 0.001 \\
Use of steroids & $1.654(0.378-7.243)$ & 0.504 \\
Resection during bronchoscopy & $4.966(1.197-20.603)$ & 0.027
\end{tabular}

$\mathrm{CT}$, computed tomography.

underwent airway intervention for their symptoms. Bronchomalacia $(28.1 \%$ vs. $75.9 \%, \mathrm{P}<0.001)$ and prior bronchoscopic airway resection $(34.4 \%$ vs. $69.0 \%, \mathrm{P}=0.007)$ were found in a larger proportion of those with recurrent symptoms compared to those without. Bronchomalacia was 17 times and prior bronchoscopic airway resection 5 times more common in patients who had recurrent symptoms after airway intervention (Table 8). A normal CT (38.7\% vs. $13.8 \%, \mathrm{P}=0.029)$ was more commonly seen among the patients who had no recurrence of symptoms after airway intervention. This association was similarly confirmed on multivariate logistic regression analysis.

\section{Discussion}

Our study findings suggest that patients who are diagnosed with TSTB secondary to previous $\mathrm{pTB}$, with recurrent lower respiratory tract infections, absence of systemic symptoms, or CT findings of fibrosis or lung collapse, may have a higher risk of symptom recurrence on conservative management alone. A normal CT is associated with reduced risk of symptom recurrence following airway intervention. Bronchomalacia and prior bronchoscopic airway resection on the other hand is associated with less favourable outcomes.

Past studies have reported a higher frequency of endobronchial tuberculosis among young females, which was postulated to be due to sociocultural expectations that discourage women from overt expectoration of phlegm, resulting in prolonged time of exposure to TB bacilli in the bronchi $(9,18)$. The same female preponderance was reflected in our study on TSTB which included nearly thrice as many women compared to men.

The higher frequency of left bronchial involvement with TSTB shown in our findings is also consistent with reports from previous studies. It is suggested that this may be related to anatomic compression by the aortic arch, more frequent TB involvement of the left sided mediastinal and hilar lymph nodes, that result in an increased risk of endobronchial infection affecting the left main bronchus $(18,19)$.

Chung et al. described 7 bronchoscopic features of endobronchial TB: non-specific bronchitic, edematoushyperemic, actively caseating, granular, tumorous, ulcerative and fibrostenotic (3). This classification essentially reflects the progression sequence of endobronchial TB. The actively caseating subtype was most frequently reported (43\%) based on this study. In contrast our findings showed a much higher proportion of fibrostenosis $(56.5 \%)$ because the majority of our patients had TSTB from previous TB rather than active disease. Also, many patients in the current study had bronchomalacia (29.0\%) and airway distortion (34.4\%). Most stenoses were long, requiring a median stent length of $40 \mathrm{~mm}$.

Factors that have been identified to increase the risk of persistent airway stenosis in patients with endobronchial TB include age greater than 45 years, presence of fibrostenotic lesions on initial bronchoscopy while on TB treatment, and long duration of symptoms lasting more than 90 days prior to start of anti-TB treatment (4). Actively caseating and edematous-hyperemic subtypes on bronchoscopy also appeared to progress to fibrostenosis more frequently compared to other subtypes (3).

Both active endobronchitis and persistent fibrostenosis that develop from previous TB may cause symptoms such as dyspnoea, wheeze and cough. Bronchoscopic intervention is a logical approach to relieve these symptoms, albeit a decision that is often made with little understanding of its outcomes, both immediate and in the longer term. The current study reported $93 \%$ of patients improved symptomatically after bronchoscopic intervention, conclusively demonstrating its effectiveness in providing immediate relief of symptoms in the majority of patients with TSTB. About half of these patients had recurrence 
of symptoms or require repeat airway intervention. The impact of airway intervention in the longer term is however less predictable. Factors associated with the recurrence of symptoms after airway intervention would be discussed in the subsequent paragraphs below.

There were significantly more asymptomatic patients in the conservatively managed group compared to the intervention group. The symptomatic patients in both groups were evaluated for factors that would predict a recurrence of symptoms or need for repeated airway intervention in the longer term.

Our analysis of the symptomatic patients who were conservatively managed, suggested that a short duration of symptoms, active TB and presence of systemic symptomlikely a reflection of disease activity-were predictive factors against symptom recurrence. This means that such patients may be expected to improve on anti-TB medication alone without the additional need for bronchoscopic interventions. Conversely, disease chronicity such as CT findings of lobar collapse or linear fibrosis, and recurrent lower respiratory tract infections were associated with higher risk of symptom recurrence if no intervention was performed.

Although these findings seem to suggest bronchoscopic intervention be considered in symptomatic patients with features of disease chronicity, the evidence from existing literature suggests otherwise. A study on CT features that predicted poor outcomes after bronchoscopic intervention showed the presence of parenchymal calcifications $(\mathrm{P}=0.002)$ and bronchiectasis $(\mathrm{P}<0.001)(20)$, markers of disease chronicity, precluded good outcomes despite airway intervention.

For patients who underwent airway intervention, the key factors associated with symptom recurrence or need for repeat airway intervention were the presence of bronchomalacia and prior bronchoscopic airway resection. We postulate that the latter might have contributed to further insult to the airway wall aggravating bronchomalacia. Patients with TSTB diagnosed on bronchoscopy, but normal CT findings were less likely to have recurrence of symptoms after a single bronchoscopic intervention.

The stent-related complication rate $(60.6 \%)$ in our study was significant, with about half of the patients requiring repeat airway intervention. This may be attributable to multiple factors including active inflammation, airway distortion, bronchomalacia, fibrosis and calcification that increase difficulty with silicone stent placement. The metallic stent is more easily deployable and conforms better to irregular stenoses. Its use in benign stenoses is however limited by concerns with difficult removal once deployed beyond a few months, and the associated complications of airway rupture \& granulomas. The advent of $3 \mathrm{D}$-printing may see future development of bespoke biodegradable stents that would hopefully circumvent some of the problems currently faced with stent insertion.

Limitations of the current study include firstly selection bias due to the retrospective nature of analysis and selection of patients from a single centre. Secondly the significant proportion of patients that were lost to follow-up (24.4\%) presented an additional source of bias to our analysis. Thirdly our interpretation of results about the outcome of steroid use in patients with TSTB was limited by the variations in dose, duration and type of steroids that were administered to patients in our cohort. Finally, our centre used only silicone stents for TSTB. Prior to the findings of the current study, it was common practice in our institution to perform silicone stent insertions, usually after balloon dilatation with or without bronchoscopic airway resection, in patients with bronchomalacia. The poor outcomes associated with inserting silicone stents in malacic airways as reflected by the high incidence (25\%) of stent migration is an important consideration because of its impact on the findings of this study. This would have contributed to the frequent symptom recurrence or need for repeat airway intervention seen in our patients with bronchomalacia.

The strengths of this study include the large number of patients and long follow-up duration compared to previous studies on TSTB (16,21-24). In addition, the analysis of outcomes for patients with TSTB who were conservatively managed provides valuable information which was lacking in older studies.

\section{Conclusions}

The presence of bronchomalacia is associated with recurrent symptoms despite airway intervention. Careful consideration should be given to weigh the benefits of immediate symptom relief with the risk of recurrent symptoms after airway intervention. Symptomatic patients with a normal CT despite bronchoscopic evidence of stenosis should be considered for airway intervention. However, bronchoscopic airway resection should be avoided. Patients who are diagnosed with TSTB early in the course of active TB treatment may do well with anti-TB medication alone. Managing TSTB is challenging, but a better understanding 
of the long-term outcomes of respective treatment options will aid physicians in their discussion with patients on the preferred choice of management.

\section{Acknowledgments}

Funding: None.

\section{Footnote}

Data Sharing Statement: Available at http://dx.doi. org/10.21037/JTD-20-670

Conflicts of Interest: All authors have completed the ICMJE uniform disclosure form (available at http://dx.doi. org/10.21037/JTD-20-670). The authors have no conflicts of interest to declare.

Ethical Statement: The authors are accountable for all aspects of the work in ensuring that questions related to the accuracy or integrity of any part of the work are appropriately investigated and resolved. The study was conducted in accordance with the Declaration of Helsinki (as revised in 2013). This was a retrospective case study. Ethics approval for this study was obtained from the SingHealth Institutional Review Board (201501-00097) without explicit consent required from the participants.

Open Access Statement: This is an Open Access article distributed in accordance with the Creative Commons Attribution-NonCommercial-NoDerivs 4.0 International License (CC BY-NC-ND 4.0), which permits the noncommercial replication and distribution of the article with the strict proviso that no changes or edits are made and the original work is properly cited (including links to both the formal publication through the relevant DOI and the license). See: https://creativecommons.org/licenses/by-nc-nd/4.0/.

\section{References}

1. So SY, Lam WK, Yu DYC. Rapid diagnosis of suspected pulmonary tuberculosis by fiberoptic bronchoscopy. Tubercle 1982;63:195-200.

2. Judd AR. Tuberculous tracheobronchitis; a study of 500 consecutive cases. J Thorac Surg 1947;16:512-23.

3. Chung HS, Lee JH. Bronchoscopic assessment of the evolution of endobronchial tuberculosis. Chest 2000;117:385-92.
4. Um SW, Yoon YS, Lee SM, et al. Predictors of persistent airway stenosis in patients with endobronchial tuberculosis. Int J Tuberc Lung Dis 2008;12:57-62.

5. Ozkaya S, Bilgin S, Findik S, et al. Endobronchial tuberculosis: Histopathological subsets and microbiological results. Multidiscip Respir Med 2012;7:34.

6. American Thoracic Society. Diagnostic standards and classification of tuberculosis. Am Rev Respir Dis 1990;142:725-35.

7. Kashyap S, Mohapatra PR, Saini V. Endobronchial tuberculosis. Indian J Chest Dis Allied Sci 2003;45:247-56.

8. Han JK, Im JG, Park JH, et al. Bronchial stenosis due to endobronchial tuberculosis: Successful treatment with self-expanding metallic stent. AJR Am J Roentgenol 1992;159:971-2.

9. Lee JH, Park SS, Lee DH, et al. Endobronchial tuberculosis. Clinical and bronchoscopic features in 121 cases. Chest 1992;102:990-4.

10. Albert RK, Petty TL. Endobronchial tuberculosis progressing to bronchial stenosis; fiberoptic bronchoscopic manifestations. Chest 1976;70:537-9.

11. Hoheisel G, Chan BK, Chan CH, et al. Endobronchial tuberculosis: diagnostic features and therapeutic outcome. Respir Med 1994;88:593-7.

12. Chan HS, Sun A, Hoheisel GB. Endobronchial tuberculosis - Is corticosteroid treatment useful? - A report of 8 cases and review of the literature. Postgrad Med J 1990;66:822-6.

13. Yaguchi D, Kimura H, Inoue N, et al. Tuberculous bronchial stenosis treated with balloon dilatation. QJM 2019;112:539-40.

14. Fang Y, You X, Sha W, et al. Bronchoscopic balloon dilatation for tuberculosis-associated tracheal stenosis: A two case report and a literature review. J Cardiothorac Surg 2016;11:21.

15. Xiong XF, Xu L, Fan LL, et al. Long-term follow-up of self-expandable metallic stents in benign tracheobronchial stenosis: A retrospective study. BMC Pulm Med 2019;19:33.

16. Low SY, Hsu A, Eng P. Interventional bronchoscopy for tuberculous tracheobronchial stenosis. Eur Respir J 2004;24:345-7.

17. Khvilivitzky K, Trivedi PN, McFadden PM. Tuberculous tracheobronchial stenosis: Avoiding resection-when less is more. J Thorac Dis 2017;9:E779-82.

18. Smart J. Endo-bronchial tuberculosis. Br J Tuberc Dis Chest 1951;45:61-8.

19. Pathak V, Shepherd RW, Shojaee S. Tracheobronchial 
tuberculosis. J Thorac Dis 2016;8:3818-25.

20. Lee JY, Yi CA, Kim TS, et al. CT scan features as predictors of patient outcome after bronchial intervention in endobronchial TB. Chest 2010;138:380-5.

21. Lim SY, Park HK, Jeon K, et al. Factors predicting outcome following airway stenting for post-tuberculosis tracheobronchial stenosis. Respirology 2011;16:959-64.

22. Iwamoto Y, Miyazawa T, Kurimoto N, et al. Interventional bronchoscopy in the management of airway stenosis due to tracheobronchial tuberculosis. Chest 2004;126:1344-52.

23. Watanabe Y, Murakami S, Oda M, et al. Treatment of bronchial stricture due to endobronchial tuberculosis. World J Surg 1997;21:480-7.

24. Ryu YJ, Kim H, Yu CM, et al. Use of silicone stents for the management of post-tuberculosis tracheobronchial stenosis. Eur Respir J 2006;28:1029-35.
Cite this article as: Lee $\mathrm{KCH}$, Tan S, Goh JK, Hsu AAL, Low SY. Long-term outcomes of tracheobronchial stenosis due to tuberculosis (TSTB) in symptomatic patients: airway intervention vs. conservative management. J Thorac Dis 2020;12(7):3640-3650. doi: 10.21037/JTD-20-670 


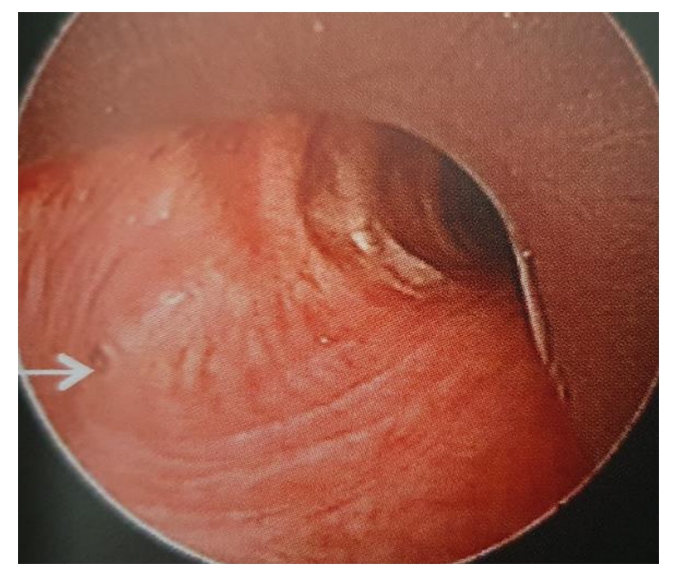

Figure S1 Pinhole stenosis (arrow) of left main bronchus (LMB) orifice (25).
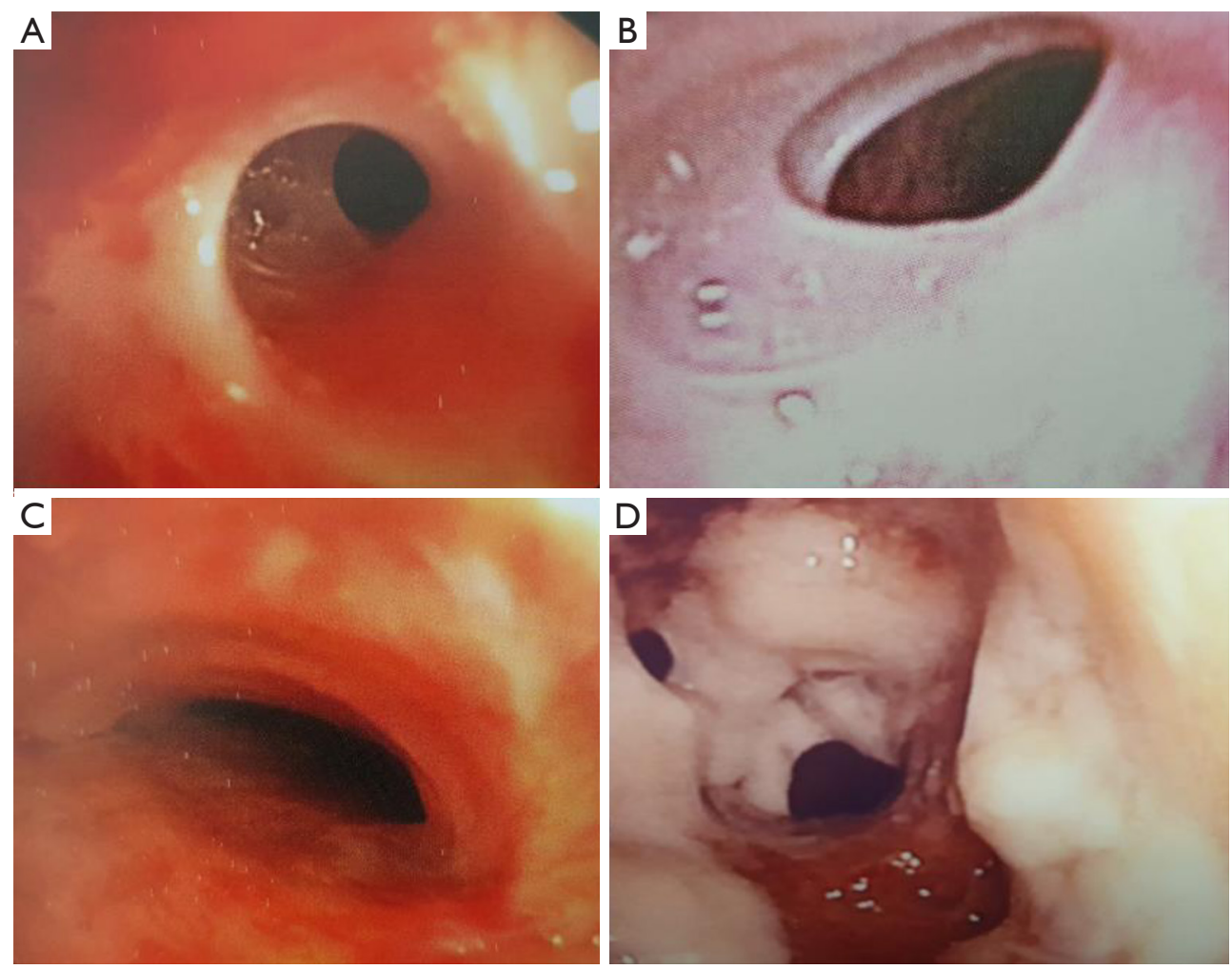

Figure S2 Endobronchial abnormalities. (A) Fibrotic stricture of right main bronchus (RMB) (25); (B) Webbed stenosis of RMB (25); (C) Tracheomalacia of the mid trachea (25); (D) Caseous material and endobronchitis of distal LMB. 


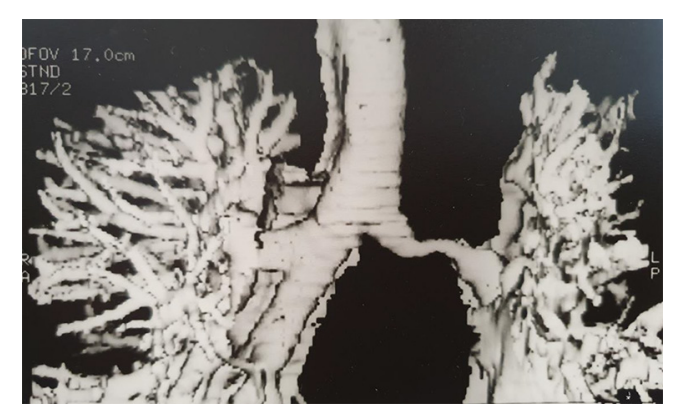

Figure S3 Distortion of the LMB evident on reconstruction computed tomography scan (26).
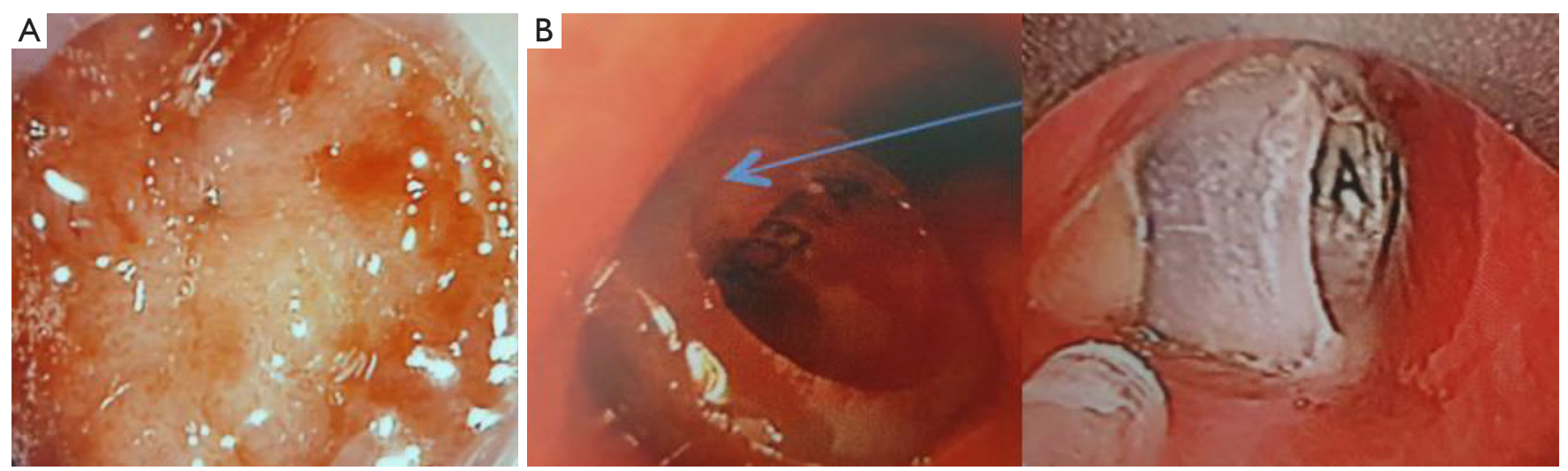

Figure S4 Stent-related complications. (A) Granuloma at distal end of silicone stent edge causing pinhole stenosis; (B) migration of LMB stent across the carina with stent edge (arrow) displaced proximally to anterior wall of distal trachea just above the carina (right picture) (25).

\section{References}

25. Hsu AAL, Lee P. Interventional bronchoscopy and pleuroscopy. A book of case studies with videos. World Scientific Publishing Co. Pte. Ltd; 2018:36,43,55-7.
26. Hsu AAL, Eng P, Ong YY. Bronchoscopic management of severe airway stenosis caused by past tuberculosis. A multimodality approach. Journal of Bronchology, 1999;6:159-65. 